\title{
Erratum to: A Trial of Rectal Indomethacin to Prevent Post-ERCP Pancreatitis in Patients with Suspected Type 3 Sphincter of Oddi Dysfunction
}

\author{
Ali A. Siddiqui ${ }^{1}$ - Devi Patel ${ }^{1} \cdot$ Jeremy Kaplan $^{1}$ - Andrew H. Zabolotsky ${ }^{1}$. \\ David Loren $^{1}$ - Thomas Kowalski ${ }^{1}$. Saad S. Ghumman ${ }^{1}$ - Douglas G. Adler ${ }^{2}$ • \\ Satish Munigala $^{3} \cdot$ Umar Hayat $^{1} \cdot$ Mohamad A. Eloubeidi $^{4}$
}

Published online: 5 October 2015

(C) Springer Science+Business Media New York 2015

\section{Erratum to: Dig Dis Sci (2015) 60:2509-2515 DOI 10.1007/s10620-015-3643-7}

In the original publication the surname of the co-author was misspelled on the initial submission. The correct spelling of name should be Satish Munigala.

Also, the authors have re-evaluated the article after publication, for the statistics on patients that had undergone a biliary sphincterotomy in their study population. It was noticed that they had erroneously miscalculated the number of patients that had undergone a biliary sphincterotomy:

1. In Table 2, the number of patients that underwent a biliary sphincterotomy in the "STENT ONLY" group is now corrected calculated as 221 and in the "Stent + Indocin" is now 36. As with the original analysis there is no statistically significant difference in the two groups (as in the original analysis)

The online version of the original article can be found under doi:10.1007/s10620-015-3643-7.

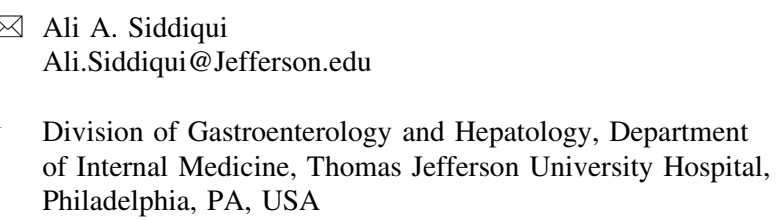

Division of Gastroenterology and Hepatology, Department of Internal Medicine, Thomas Jefferson University Hospital, Philadelphia, PA, USA

2 Division of Gastroenterology and Hepatology, Department of Internal Medicine, University of Utah, Salt Lake City, UT, USA

3 Department of Internal Medicine, Washington University School of Medicine, St. Louis, MO, USA

4 Division of Gastroenterology, Northeast Alabama Regional Medical Center, Anniston, AL, USA
2. Tables 3 and 4 have now undergone a similar analysis with the correct number of patients who had undergone a biliary sphincterotomy. Again, there was no statistically significant difference in the two groups (as in our original analysis)

3. In essence, the re-calculated data on biliary sphincterotomy does not change any conclusions in the paper

In the Results section, the sentence "Stepwise multiple logistic regression was performed on 5 potential risk factors in the univariate analysis" should be deleted. Edits made to the draft manuscript using MS Word's track changes were not visible in the final file, and thus erroneously appeared in the published article. 